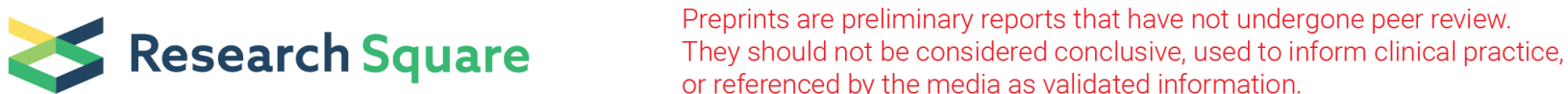

\section{Safety review of 42 cases of COVID-19 treated with low- dose chloroquine}

\section{Bo Zhou}

Department of Pharmacy, Guangzhou Eighth People's Hospital, Guangzhou Medical University, Guangzhou 510060, China https://orcid.org/0000-0002-9334-1124

\section{Jing Zhang}

Department of Pharmacy, Guangzhou Eighth People's Hospital, Guangzhou Medical University, Guangzhou 510060, China

\section{Yanqing Liu}

Department of Pharmacy, Guangzhou Eighth People's Hospital, Guangzhou Medical University, Guangzhou 510060, China

\section{Wenxin Hong}

Infectious Disease Center, Guangzhou Eighth People's Hospital, Guangzhou Medical University, Guangzhou, China

\section{Fengbi Jian}

Department of Pharmacy, Guangzhou Eighth People's Hospital, Guangzhou Medical University, Guangzhou 510060, China

\section{Ming Wang}

Department of Pharmacy, Guangzhou Eighth People's Hospital, Guangzhou Medical University, Guangzhou 510060, China

\section{Jun Tan}

Department of Pharmacy, Guangzhou Eighth People's Hospital, Guangzhou Medical University, Guangzhou 510060, China

\section{Weiping Cai}

Infectious Disease Center, Guangzhou Eighth People's Hospital, Guangzhou Medical University, Guangzhou, China

\section{Fuchun Zhang}

Infectious Disease Center, Guangzhou Eighth People's Hospital, Guangzhou Medical University, Guangzhou, China

\section{Linghua Li ( $\square$ llheliza@126.com )}

Infectious Disease Center, Guangzhou Eighth People's Hospital, Guangzhou Medical University, Guangzhou, China

\section{Research}

Keywords: COVID-19, Chloroquine, Safety, Adverse reactions

Posted Date: April 24th, 2020

DOI: https://doi.org/10.21203/rs.3.rs-22918/v1

License: (c) (i) This work is licensed under a Creative Commons Attribution 4.0 International License. Read Full License 


\section{Abstract}

\section{Background}

Coronavirus Disease 2019 (COVID-19) has become a global pandemic and caused over one hundred thousand death. Chloroquine (CQ) and hydroxychloroquine (HCQ) were recommended for off-label use in the treatment of COVID-19 in some countries despite their unclear benefit. However, the toxicity of these agents has been ignored, so the investigation of their safety in the treatment of COVID-19 is crucial for providing a reference for the rational use.

\section{Methods}

The medical records obtained from the information management system of Guangzhou Eighth People's Hospital were reviewed to extract data about patients who received chloroquine phosphate tablets for COVID-19 treatment from January 20th to March 5th, 2020. The data were assessed to determine the correlation of adverse reaction with chloroquine phosphate based on Chinese CFDA standards as well as the severity of adverse events based on American CTCAE5.0 standard, and evaluate the safety of this medication.

\section{Results}

A total of 42 patients (23 males and 19 females, average $42.19 \pm 14.29$ years old) with COVID-19 were treated with lowdose chloroquine phosphate (oral, $500 \mathrm{mg}$, once per day). Totally 18 patients $₫ 42.86 \%$ 囚experienced 20 adverse events. The mean duration of CQ administration was 6.57 days (SD, 3.16 days; range: 1 day to 16 days) and $52.4 \%$ received CQ for 7 to 9 days. The adverse events occurred within 6-8 days of treatment. For the 20 adverse events, 19 were not higher than grade 2 and only one was grade 3 because of the severely limited self-care ability in one patient. The most common adverse events were related to the digestive, circulatory, hepatic, and nervous systems.

\section{Conclusion}

Oral chloroquine phosphate tablets resulted in a high incidence of adverse reactions. In the clinical trial of chloroquine phosphate for COVID-19 treatment, it should be used under pharmaceutical care; timely evaluation of the drug safety during the treatment process is necessary and a randomized controlled clinical trial should be conducted.

\section{Introduction}

The Coronavirus Disease 2019 (COVID-19) infection started in Hubei province in December 2019 and has spread rapidly. It is an acute respiratory infection that is treated as a Class $A$ infectious disease. In China, various drugs have been tested for treating COVID-19, but all of them are off-label. Recently, chloroquine has been added as a trial drug option in the sixth edition of the COVID-19 medical protocol [1] (hereinafter referred to as the medical protocol). According to the sixth edition, the recommended dose for COVID-19 is 500 mg for adults, twice a day, for a maximum of 10 days. However, chloroquine has multiple and frequent adverse effects, and its use for COVID-19 treatment therefore warrants safety evaluation. The national health board subsequently issued a notice to adjust the usage and dosage of chloroquine phosphate and the applicable target population for COVID-19 treatment [2], and the changes were published in the seventh edition of the medical protocol [3]. Guangzhou No.8 People's Hospital (hereinafter referred to as 'our hospital') is one of the designated hospitals for COVID-19 treatment where the use of chloroquine phosphate has been tested as an antiviral therapy. Given its safety concerns, in the seventh edition, the treatment regimen for adults was changed to $500 \mathrm{mg}$ once a day instead of the previous twice a day. 


\section{Methods}

\section{General information}

\section{Source and grouping of cases}

We reviewed the medical records of COVID-19 patients admitted to our hospital from January 20th 2020 to March 5th, 2020. Inclusion criteria: patients diagnosed with COVID-19 who met the diagnostic criteria for confirmed cases in the sixth edition of the medical protocol(1) [1]; were treated with low-dose chloroquine phosphate (oral, 500 mg, once a day) during their hospitalisation; and did not use more than two kinds of antiviral drugs for 2 days before and after the treatment period. The exclusion criterion was that existing an unreasonable combination of drugs determined by the clinical pharmacist [4] or in line with the drug use contraindications. There are forty-two patients met the criteria.

\section{Source of chloroquine phosphate is shown in Table 1.}

Table 1. Source of chloroquine phosphate

\begin{tabular}{lllll} 
Number & Medicine name & Specification & Factory & $\begin{array}{l}\text { Lot } \\
\text { number }\end{array}$ \\
\hline $\mathbf{1}$ & $\begin{array}{l}\text { Chloroquine phosphate } \\
\text { tablets }\end{array}$ & $0.25 \mathrm{~g} * 8 /$ board & Shanghai Zhongxi Pharmaceutical Co., Ltd. & 180601 \\
\hline $\mathbf{2}$ & $\begin{array}{l}\text { Chloroquine phosphate } \\
\text { tablets }\end{array}$ & $0.25 \mathrm{~g} * 8 / \mathrm{box}$ & $\begin{array}{l}\text { Shanghai Xinyi Tianping Pharmaceutical } \\
\text { Co., Ltd. }\end{array}$ & 12181101 \\
\hline $\mathbf{3}$ & $\begin{array}{l}\text { Chloroquine phosphate } \\
\text { tablets }\end{array}$ & $0.25 * 40 /$ bottle & $\begin{array}{l}\text { Guangdong Zhongsheng Pharmaceutical } \\
\text { Trading Co., Ltd. }\end{array}$ & 200205 \\
& & & 0
\end{tabular}

\section{Methods}

\section{Medical information collection methods}

This study followed a retrospective design wherein the COVID-19 patient records were obtained through the hospital information management system. Specifically, the records of patients who received chloroquine phosphate tablets were evaluated by the clinical pharmacists to extract relevant data, including patient record number, sex, age, disease classification, medical history, history of allergies, drug information, adverse reactions, drug combinations, adverse reaction processing and other related content, with the aim of understanding the actual situation of adverse drug reactions caused by chloroquine phosphate during COVID-19 treatment.

\section{Method of administration}

For COVID-19 treatment, 500 mg chloroquine phosphate tablets were administered orally once a day. The observation period for adverse events was from the day before the administration to the fifth day after the cessation of chloroquine administration.

\section{Statistical analysis}

SPSS16.0 software was used for the statistical analyses. The magnitude is presented as the mean \pm standard deviation (SD), and the incidence is presented as the percentage (\%). 


\section{Drug safety}

According to the evaluation criteria for common adverse events proposed by the US Department of Health and Human Services (CTCAE5.0) [5], adverse events were classified into five grades. A lower incidence of adverse events in patients after treatment indicates a level of higher drug safety.

\section{Evaluation of the association between adverse events and chloroquine phosphate}

The associations of adverse events with chloroquine phosphate were assessed according to the six categories formulated by the national monitoring centre for adverse drug reactions: affirmative, probable, possible, unlikely, unevaluated and unassessed. The associations classified as positive, probable and possible adverse events were considered as adverse events $[6,7]$. The adverse events were then classified according to the involved organs and systems based on CTCAE5.0.

\section{Results}

Clinical use of chloroquine phosphate

Demographic status is shown in Table 2.

Table 2. Demographic indicator

\begin{tabular}{|llll|}
\hline & Gender(male/female) & Age (years) & Average length of stay duration (days) \\
\hline Patients & $23 / 19$ & $42.19 \pm 14.29$ & $19.07 \pm 7.65$ \\
\hline
\end{tabular}

\section{COVID-19 severity and underlying diseases}

Among the 42 patients included in the study, none were critical case, 3 (7.14\%) were mild ones, $36(85.72 \%)$ were moderate ones and $3(7.14 \%)$ were severe ones. Nine (21.43\%) patients had at least one underlying diseases, including hypertension (6 patients), hepatitis B (2 patients), hypothyroidism (1 patient), gout (1 patient), deafness (1 patient) and asthma (1 patient).

\section{Previous history of allergy and medication}

Among the 42 patients who received low-dose chloroquine phosphate, 3 had a history of allergy, including 1 patient with penicillin allergy and 2 patients with cephalosporin allergy.

\section{Treatment course}

The mean duration of chloroquine phosphate administration was 6.57 days (SD, 3.16 days; range: 1 day to 16 days), and $52.4 \%$ of the patients received treatment 7-9 days.

\section{Drug combinations}

Up to 5 March, chloroquine phosphate for COVID-19 treatment was tested in 42 patients. The medication status of the patients is shown in Table 3. The mean number of drug varieties that the patients were receiving simultaneously was 
6.93 (SD, 5.38; range: 2-27). Of the 42 patients, 33 were receiving Chinese herbal medicine and 35 were receiving antibiotics simultaneously.

There were no cases in which three or more kinds of drugs were tried for antiviral treatment. Considering the therapeutic treatment course (as mentioned in 2.1.4) and drug combinations, the medication statuses of patients were significantly different. The patients' medical records were reviewed by clinical pharmacists to assess and record the risks of all medications. In one patient, chloroquine phosphate was discontinued after day 1 of the trial due to the change in the medical protocol and was resumed after 8 days. Overall, the medication administration was in accordance with the diagnosis and treatment protocol prescribed at the corresponding time (sixth or seventh version).

Table 3. Clinical trial of chloroquine phosphate

\begin{tabular}{|lllll|}
\hline & $\begin{array}{l}\text { Number of } \\
\text { cases }\end{array}$ & $\begin{array}{l}\text { Gender } \\
\text { (male/female) }\end{array}$ & $\begin{array}{l}\text { Number of drug } \\
\text { varieties * }\end{array}$ & $\begin{array}{l}\text { Type } \\
\text { composition \# }\end{array}$ \\
\hline Chloroquine alone & 22 & $11 / 11$ & $7.05 \pm 6.00$ & $2 / 17 / 3 / 0$ \\
\hline $\begin{array}{l}\text { Chloroquine with kaletra } \\
\text { Chloroquine with }\end{array}$ & 9 & $5 / 4$ & $7.00 \pm 5.31$ & $0 / 9 / 0 / 0$ \\
$\begin{array}{l}\text { arbidol } \\
\begin{array}{l}\text { Chloroquine with antiviral } \\
\text { agents }\end{array}\end{array}$ & 0 & $7 / 4$ & $6.64 \pm 3.89$ & $1 / 10 / 0 / 0$ \\
\hline \begin{tabular}{l} 
Summary \\
\hline
\end{tabular} & 42 & 0 & 0 & 0 \\
\hline
\end{tabular}

* Number of drug varieties during and before and after chloroquine administration (excluding traditional Chinese medicine)

\# Type composition is mild/ ordinary /severe/ critical in turn

\section{Occurrence of adverse reactions}

\section{Adverse events in this clinical trial}

The data on adverse events were extracted from the medical records. Two cases of adverse events were found during follow-up by clinical pharmacists and stool $\geq 3$ times a day was recorded as diarrhoea.

Among the 42 patients receiving low-dose chloroquine phosphate, 22 had suspected adverse events related to chloroquine phosphate, and the correlation was evaluated according to the standards developed by the national monitoring centre for adverse drug reactions. The evaluation results were as follows: the correlation was positive in 3 patients, probable in 5, possible in 12, and irrelevant in 2, giving a total of 20 adverse events in 18 patients(42.86\%). The classification and clinical manifestations of adverse events are listed in Table 4. The adverse events were graded according to the US CTCAE, and the results are shown in Table 5.

A severe clinical manifestation of adverse event was observed. One patient developed a mental disorder on the afternoon of the eighth day of treatment, showed abnormal mental behaviour, complaining of being monitored and becoming fidgety. The patient stopped taking chloroquine phosphate the next day and received symptomatic treatment. The delusions and hyperactivity disappeared on the third day. 
In addition, there was another typical manifestation of adverse event, but it was grade 2. A patient with abdominal pain and diarrhoea, excluding or considering the effect of the combination of drugs, did not show improvement until 4 days after the cessation of chloroquine administration. Patients with other adverse events for digestive system such as gastric uneasiness were given medicine to relieve belching and bloating. Tachycardia and arrhythmia in patients with heart disease were assessed by electrocardiogram (ECG) examination, but a contrast for this assessment wasn't conducted before and after treatment with chloroquine phosphate, which may lead to misjudgement. The liver function of patients was assessed in accordance with the criteria for liver function impairment reported in the CTCAE.

Table 4. Classification and clinical manifestations of chloroquine phosphate adverse reactions

\begin{tabular}{|lll|}
\hline Classification and clinical manifestations of adverse reactions & Cases & Incidence rate \\
\hline Digestive system & 12 & $28.57 \%$ \\
\hline Diarrhea and abdominal pain & 3 & \\
\hline Nausea, vomiting, loss of appetite & 4 & \\
\hline Other stomach discomfort & 4 & \\
\hline Cardiac system & 5 & $11.90 \%$ \\
\hline Bradycardia & 2 & \\
\hline Arrhythmia & 2 & \\
\hline Myocardial damage & 1 & \\
\hline Liver system & 2 & $4.76 \%$ \\
\hline Liver damage & 2 & \\
\hline Mental illness & 1 & $2.38 \%$ \\
\hline Anxiety delusion & 1 & \\
\hline
\end{tabular}

In this retrospective analysis, we found that the incidence of adverse events related to chloroquine phosphate was high: 13 patients had grade 2 adverse events (affecting daily activities), and 1 patient had grade 3 adverse event (severe symptoms with limited self-care ability).

Table 5. Classification of adverse events of chloroquine phosphate

\begin{tabular}{|llllll|}
\hline & Grade 1 & Grade 2 & Grade 3 & Grade 4 & Grade 5 \\
\hline Occurrences & 6 & 13 & 1 & 0 & 0 \\
\hline Proportion & $30 \%$ & $65 \%$ & $5 \%$ & $0 \%$ & $0 \%$ \\
\hline
\end{tabular}

\section{The influence of dosage and course of treatment}

All 42 patients were orally administered $500 \mathrm{mg}$ chloroquine phosphate once a day. The associations of adverse events and their time of occurrence with the course of treatment are described in Tables 6 and 7.

Table 6. Trial course of chloroquine phosphate and number of corresponding adverse reactions 


\begin{tabular}{|llll|}
\hline Course of treatment & Number of cases & Constituent ratio & Number of adverse reactions \\
\hline $\mathbf{S}$ 2 days & 7 & $16.67 \%$ & 3 \\
\hline 3-6 days & 9 & $21.43 \%$ & 5 \\
\hline 7-9 days & 20 & $47.62 \%$ & 11 \\
\hline 10 days $\leq$ & 6 & $14.28 \%$ & 1 \\
\hline
\end{tabular}

Table 7. Time of occurrence of adverse events to chloroquine phosphate

\begin{tabular}{|lll|}
\hline Time of occurrence & Number of cases & Adverse reaction classification \# \\
\hline Day 1 & 1 & $1 / 0 / 0$ \\
\hline Day 2 & 2 & $0 / 2 / 0$ \\
\hline Day 3 & 2 & $2 / 0 / 0$ \\
\hline Day 4 & 2 & $1 / 1 / 0$ \\
\hline Day 6 & 4 & $1 / 3 / 0$ \\
\hline Day 7 & 4 & $0 / 4 / 0$ \\
\hline Day 8 & 3 & $1 / 2 / 0$ \\
\hline Day 9 & 1 & $0 / 1 / 0$ \\
\hline Day 10 & 1 & $0 / 0 / 1$ \\
\hline
\end{tabular}

\# Note: Adverse reaction classification is grade1 / grade 2 / grade 3 in turn

As shown in Tables 6 and 7, nearly half of the 42 patients received chloroquine phosphate for 7-9 days, and the incidence of adverse events corresponded to this duration. In patients whose treatment course lasted $\geq 10$ days, the incidence of adverse events was low, probably because they had good tolerance or the observation days were insufficient to notice the effects of drug accumulation. The occurrence time of adverse events was relatively concentrated on the sixth to eight days of the treatment course, as shown in Table 3.

According to the above two Tables 6 and 7, the incidence of adverse events was more than $50 \%$ among patients whose treatment course lasted $<10$ days. According to the pharmacokinetic data, $500 \mathrm{mg}$ chloroquine phosphate can accumulate in vivo when administered for more than 7 days. Patients whose treatment course lasts $\geq 6$ days may develop liver damage or psychiatric symptoms due to drug accumulation, and most of these adverse events require drug withdrawal and symptomatic treatment. According to the literature, even if a patient tolerates chloroquine phosphate well, he/she may subsequently develop liver damage, psychiatric symptoms, cardiotoxicity, eye problems, etc.

\section{The influence of drug using}

The specific adverse events of chloroquine phosphate when administered in combination with other drugs are shown in Table 8.

Table 8. Number of adverse events occurred with chloroquine phosphate and other antiviral drugs 


\begin{tabular}{|lllll|}
\hline $\begin{array}{l}\text { Medication } \\
\text { status }\end{array}$ & $\begin{array}{l}\text { Number of } \\
\text { cases }\end{array}$ & $\begin{array}{l}\text { Proportion of } \\
\text { cases }\end{array}$ & $\begin{array}{l}\text { Number of adverse } \\
\text { reactions }\end{array}$ & $\begin{array}{l}\text { Proportion of adverse } \\
\text { reactions }\end{array}$ \\
\hline $\begin{array}{l}\text { Chloroquine alone } \\
\text { Chloroquine with }\end{array}$ & 22 & $52.38 \%$ & 8 & $40 \%$ \\
kaletra & 9 & $21.43 \%$ & 8 & $40 \%$ \\
\hline $\begin{array}{l}\text { Chloroquine with } \\
\text { arbidol }\end{array}$ & 11 & $26.19 \%$ & 4 & $20 \%$ \\
\hline
\end{tabular}

Among the 9 patients who received chloroquine phosphate combined with Kaletra antiviral treatment, 6 received sequential treatment, with Kaletra prior to chloroquine phosphate. Among the 11 patients who received chloroquine phosphate combined with abidol antiviral treatment, 4 received sequential treatment, with abidol prior to chloroquine phosphate. This finding shows that the incidence of adverse events of chloroquine combined with Kaletra is relatively high, and these adverse events are related to not only to the digestive system but also to the cardiac and hepatic systems.

In this retrospective analysis, we found that 42 COVID-19 patients received chloroquine, 9 did not receive antiviral treatment during the same period, and 155 received other antiviral drugs during the same period. The details of these treatments are shown in Table 9. Although the incidence of adverse events was not significantly different between these treatment groups, the incidence of adverse events was higher with chloroquine than with other treatments.

Table 9. Comparison of adverse events between patients trying chloroquine phosphate and other patients

\begin{tabular}{|lll|}
\hline & $\begin{array}{l}\text { Number of adverse events } \\
\text { occurred }\end{array}$ & $\begin{array}{l}\text { Number of adverse events not } \\
\text { occurred }\end{array}$ \\
\hline No antiviral agents & 2 & 7 \\
\hline $\begin{array}{l}\text { Patients who try chloroquine } \\
\text { phosphate }\end{array}$ & 18 & 24 \\
\hline $\begin{array}{l}\text { Patients who try other antiviral } \\
\text { agents }\end{array}$ & 48 & 107 \\
\hline
\end{tabular}

\section{Risk factors for adverse reactions}

Comprehensive clinical records and supporting materials, because various factors do not show a normal distribution and there are many factors related to adverse events caused by chloroquine phosphate, we use chi-square test. We evaluated the influence of factors such as underlying disease type, sex, age, comorbidity, drug combinations, and allergy history on the incidence of adverse events caused by chloroquine phosphate using factor analysis, and the results are shown in Table 10.

Table 10. Analysis of influencing factors of adverse events related to chloroquine phosphate(n[\%]) 


\begin{tabular}{|c|c|c|c|}
\hline & Occurrence group $(n=18)$ & No occurrence group( $(n=24)$ & $P$ \\
\hline \multicolumn{4}{|l|}{ Gender } \\
\hline Male & $13(72.2)$ & $10(41.7)$ & \multirow[t]{2}{*}{0.049} \\
\hline Female & $5(27.8)$ & $14(58.3)$ & \\
\hline \multicolumn{4}{|l|}{ Age } \\
\hline$<50$ years old & $11(61.1)$ & $16(66.7)$ & \multirow[t]{2}{*}{0.710} \\
\hline$>50$ years old & $7(38.9)$ & 8 (33.3) & \\
\hline \multicolumn{4}{|l|}{ Clinical type } \\
\hline Mild & $2(11.1)$ & $1(4.2)$ & \multirow[t]{3}{*}{0.445} \\
\hline Ordinary & $14(77.8)$ & $22(91.7)$ & \\
\hline Severe & $2(11.1)$ & $1(4.2)$ & \\
\hline \multicolumn{4}{|l|}{ Comorbidity } \\
\hline Yes & $5(27.8)$ & $4(16.7)$ & \multirow[t]{2}{*}{0.625} \\
\hline No & $13(72.2)$ & $20(83.3)$ & \\
\hline \multicolumn{4}{|c|}{ Combination of drugs } \\
\hline Yes & $10(55.6)$ & $10(41.7)$ & \multirow[t]{2}{*}{0.372} \\
\hline No & $8(44.4)$ & $14(58.3)$ & \\
\hline \multicolumn{4}{|l|}{ Allergic history } \\
\hline Yes & $1(5.6)$ & $2(8.3)$ & \multirow[t]{2}{*}{1.000} \\
\hline No & $17(94.4)$ & $22(91.7)$ & \\
\hline
\end{tabular}

The findings showed that the proportion of male patients in the occurrence group (patients who showed adverse reactions) was higher than that in the no occurrence group (patients who showed no adverse reactions) $(P<0.05)$. However, no statistically significant difference was found between the two groups in terms of age, underlying disease type, comorbidity, drug combinations or allergy history $(P>0.05)$.

\section{Discussion}

Chloroquine phosphate, a classic anti-malarial drug, was added as a choice of antiviral drugs in the COVID-19 treatment programme (trial version 6). The common adverse events to chloroquine phosphate are vision problems. Prolonged use of chloroquine phosphate can affect the vision, often irreversible, and can also damage hearing and cause sinus node dysfunction [4]. In addition to malaria and COVID-19, chloroquine phosphate has been used in the treatment of lupus erythematosus because of its immunomodulatory function. None of the patients on the clinical trial of chloroquine phosphate for COVID-19 had any contraindication for chloroquine, but most of the patients did not undergo G6PD examination before medication for chloroquine was forbidden to G6PD lacking patients. During medication, if intolerable toxic or side effects were observed in any patient, chloroquine phosphate administration was stopped. 
Trial version 6 of the recommended treatment regimen involved $500 \mathrm{mg}$ chloroquine phosphate twice a day for up to 10 days. A National Health Commission of the People's Republic of China dose adjustment notice was issued 8 days later [3] that entailed usage and dosage adjustment for drug safety concerns. Accordingly, the updated usage and dosage requirements in the trial version 7 were as follows: for 18-65-year-old patients weighing greater than $50 \mathrm{~kg}$, $500 \mathrm{mg}$ twice a day for 7 days, and for those weighing less than $50 \mathrm{~kg}, 500 \mathrm{mg}$ twice a day on the first and second days and once a day from the third to seventh day.

According to the WHO and US malaria guidelines $[8,9]$, the recommended regimen and dosage for uncomplicated malaria are 1000-mg chloroquine phosphate taken immediately, and 500 mg chloroquine phosphate taken after 6, 24 and $48 \mathrm{~h}$, giving a total dose of $2500 \mathrm{mg}$ chloroquine phosphate. Signs of adverse reactions, but no headache or rash, were found in this study.

For the treatment of malaria-causing Plasmodium vivax and $P$. malariae infections in China [10,11], the use of chloroquine and primaquine is recommended, with the total administrable oral dose of chloroquine limited to 1200 $\mathrm{mg}$. The specific dosage is $600 \mathrm{mg}$ once or $300 \mathrm{mg}$ twice on day 1, followed by $300 \mathrm{mg}$ once daily on days 2 and 3 .

Although there are limited instructions for the trial use of chloroquine phosphate for COVID-19 treatment, there is a basis for the selection of this drug and its dosage. For example, chloroquine phosphate is contraindicated in patients with liver and kidney dysfunction, heart disease, severe pleomorphic erythema, hematoporphyria, psoriasis or psychosis. Our review of the medical records showed no inappropriate selection of chloroquine phosphate in contraindicated cases.

Chloroquine is mainly metabolised in the liver by hepatic drug-metabolising enzymes CYP2C8, CYP3A4 and CYP2D6 [12]. The drug lopinavir/ritonavir can inhibit CYP2C8, CYP3A4 and CYP2D6 activities, resulting in increased plasma chloroquine concentrations. However, due to the lack of data on the combination of these drugs with chloroquine, no reliable dosage adjustment recommendation has been issued. During the treatment, nine patients were treated with chloroquine combined with lopinavir/ritonavir, six of whom were treated with sequential therapy with at least a 1-day interval.

Chloroquine phosphate combined with moxifloxacin, azithromycin or clarithromycin may increase the risk of arrhythmias $[13,14,15,16]$. Our study showed that chloroquine phosphate was given in combination with moxifloxacin in 14 patients for COVID-19 treatment. Taken together, these finding indicate that some risky combinations of drugs were used in our retrospectively analysed cases. In addition, one patient had arrhythmia, so treatment with chloroquine phosphate was terminated in this case. In the trial of chloroquine phosphate, risk assessment is essential, including the assessment of underlying diseases, interactions, and adverse reactions, to reduce the risk of cardiotoxicity and chloroquine accumulation.

Except for one case of psychiatric disorder, all other adverse events were mild, and no significant difference was observed in the incidence of adverse events between various allergic constitutions. Compared with other medication programs, treatment with chloroquine phosphate did not increase the risk of disease progression and mortality, so it can be considered tolerable. However, the treatment is associated with a high incidence of adverse reactions, which should be prevented. Given the limitation of the retrospective nature of this study, the conclusions need to be verified in a high-quality randomised control trial with a large sample size.

In the early stage of antiviral treatment, the usage and dosage of chloroquine phosphate were adjusted in consideration of drug safety and pharmacokinetics. Clinical pharmacists were involved in the recording of haematologic, hepatic and renal functions and blood coagulation indicators to analyse the rationality of combined 
drug use and prevent and reduce the use of risky drug combinations. Chloroquine concentrations in the plasma were also monitored to assess the treatment requirements and reduce chloroquine accumulation, and to assess the metabolisation and elimination of chloroquine.

In this retrospective study, the patients were subject to centralized observation, so the patient records and treatment information of adverse events were relatively complete. The data were true and reliable and allowed a statistical analysis of the incidence of adverse events caused by chloroquine phosphate in a specific population over a specific period. However, there were some limitations. First, because the observation time of adverse events was short, the drug accumulation in patients may not have completely cleared, and some adverse effects would have occurred after the observation period, which indicates a possible risk of omission. Second, ECG examinations were not performed at baseline, so related adverse events could be misjudged. Third, some rare or mild adverse events that are difficult to evaluate may have gone unrecorded. Fourth, chloroquine phosphate tablets from three manufacturers were used, but we did not perform a manufacturer analysis.

\section{Conclusions}

Our retrospective analysis revealed that $17.35 \%$ of the COVID-19 patients in our hospital received chloroquine phosphate treatment (as of 29 February), and the incidence of adverse events was $42.86 \%$ (as of 5 March). These reactions were mainly related to the digestive, cardiac and hepatic systems, with one case of psychiatric disorder. Notably, $65 \%$ of the adverse events were grade 2 . No life-threatening, lethal or teratogenic disability cases were found, indicating that chloroquine phosphate is relatively safe for COVID-19 treatment. The indications for using chloroquine phosphate tablets for COVID-19 are based on clinical trials, and the usage and dosage regulations are closer to the seventh edition of the medical protocol[3] except for a smaller dosage. There are differences between the course of medication and various diagnosis and treatment programmes and guidelines. The adverse events to chloroquine phosphate tablets were compared with the data of 205 COVID-19 patients discharged from hospital from 21 January to 25 February. Chi-square tests showed that the incidence of adverse events caused by chloroquine phosphate was high, but this incidence was not significantly different from those caused by other antiviral agents. Risk factor analysis showed that sex was the only significant factor affecting the incidence of adverse events related to chloroquine phosphate.

Given the high incidence of adverse reactions, clinical trials of chloroquine phosphate for COVID-19 treatment should take into consideration the abovementioned risk factors when evaluating the drug's safety, in addition to other factors such as length of stay, days of positive-to-negative conversion, rate of negative-to-positive conversion when discharged, etc. Specific population groups should be monitored intensively, and ECG examinations of patients should be performed at baseline. Furthermore, clinical pharmacists should intervene and monitor medication as early as possible to avoid inappropriate medication.

\section{Abbreviations}

COVID-19: Coronavirus Disease 2019

SPSS: Statistical product and service solutions

SD: standard deviation

CTCAE: Common Terminology Criteria for Adverse Events

Page $11 / 13$ 
ECG: electrocardiogram

\section{Declarations}

\section{Acknowledgements}

We thank all patients who participated in this study and all staff of Guangzhou Eighth People's Hospital for their clinical care given to patients and facilitating access to the relevant medical records.

\section{Authors' contributions}

ZB undertook the most of the work, including adverse reaction judgment, pharmaceutical monitoring and manuscript writing. ZJ, LY, JF and WM participated in data collection and analysis. HW, TJ and CW provided clinical guidance and improve the clinical trial. ZF was the project sponsor. And LL, as s principal author, participated in coordination, clinical observation and manuscript writing. All authors read and approved the final manuscript.

\section{Funding}

This study was supported by Chinese 13th Five-Year National Science and technology major project 『2018ZX10302103-002, 2017ZX10202102-003-004囚, and Infectious Disease Specialty of Guangzhou High-level Clinical Key Specialty (2019-2021).

\section{Availability of data and materials}

The datasets used and/or analysed during the current study are available from the corresponding author on reasonable request.

\section{Ethics approval and consent to participate}

Ethics Committee of Guangzhou Eighth People's Hospital approved this study.

\section{Consent for publication}

Not applicable

\section{Competing interests}

The authors declare that they have no competing interests.

\section{References}

1. General Office of the National Health Commission of People's Republic of China. Notice on printing and distributing Diagnosis and Treatment Plan for COVID-19 (Trial Version 6). 2020.

http://www.nhc.gov.cn/yzygj/s7653p/202002/8334a8326dd94d329df351d7da8aefc2.shtml.

2. General Office of the National Health Commission of People's Republic of China. Notice on adjusting the usage and dosage of trial chloroquine Phosphate in the treatment of COVID-19. 2020.http://www.nhc.gov.cn/yzygj/s7653p/202002/0293d017621941f6b2a4890035243730.shtml.

3. General Office of the National Health Commission of People's Republic of China. Notice on printing and distributing Diagnosis and Treatment Plan for COVID-19 (Trial Version7). 2020. 
http://www.nhc.gov.cn/yzygj/s7653p/202003/46c9294a7dfe4cef80dc7f5912eb1989.shtml.

4. Instructions for chloroquine phosphate tablets. https://db.yaozh.com/instruct/174397.html

5. U.S. DEPARTMENT OF HEALTH AND HUMAN SERVICES: Common Terminology Criteria for Adverse Events (CTCAE) Version 5.0. 2017.

https://ctep.cancer.gov/protocoldevelopment/electronic_applications/docs/CTCAE_v5_Quick_Reference_5x7.pdf.

6. National Adverse Drug Reaction Monitoring Center. Adverse drug reaction reporting and monitoring workbook. 2005.

7. The Minister of Health of the People冈s Republic of China: Measures for the administration of reporting and monitoring of adverse drug reactions. 2012.

http://www.nhc.gov.cn/wjw/bmgz/201105/b442a66fc52b4793a57160002ac2a1a9.shtml.

8. WHO: GUIDELINES FOR THE TREATMENT OF MALARIA Third edition. 2015.

https://www.who.int/malaria/publications/atoz/9789241549127/en.

9. U.S. CENTERS FOR DISEASE CONTROL AND PREVENTION: Treatment of Malaria (Guidelines for Clinicians). 2013. https://www.cdc.gov/malaria/diagnosis_treatment/treatment.html.

10. The general office of the Ministry of Health of the People's Republic of China. The general office of the Ministry of Health of the People's Republic of China issuance the notice of the revised draft of the principles and drug regimen for the use of antimalarial drugs. 2009.

http://www.nhc.gov.cn/bgt/s9512/200907/7e3faa5faad4483b8305917c1ec9027d.shtml.

11. National Health and Family Planning Commission of the People's Republic of China: WS/T 485-2016 Specification for the use of antimalarial drugs. 2016.

http://www.nhc.gov.cn/wjw/s9499/201605/68001801f8af435bba987b84891aefc6.shtml

12. Denis Projean, Bruno Baune, et al. In vitro metabolism of chloroquine: identification of CYP2C8, CYP3A4, and CYP2D6 as the main isoforms catalyzing N-desethylchloroquine formation. Drug Metab. Dispos. 2003; 31(6):74854.

13. Gopi Krishna Panicker, Dilip R Karnad, Pramod Kadam, et al. Detecting moxifloxacin-induced QTc prolongation in thorough QT and early clinical phase studies using a highly automated ECG analysis approach. Br. J. Pharmacol. 2016; 173: 1373-80.

14. Sean N. Avedissian, Nathaniel J. Rhodes, Tien M.H. Ng, et al. The Potential for QT Interval Prolongation with Chronic Azithromycin Therapy in Adult Cystic Fibrosis Patients. Pharmacotherapy. 2019; 39(6):718-23

15. Niedrig David, Maechler Sarah, Hoppe Liesa, et al. Drug safety of macrolide and quinolone antibiotics in a tertiary care hospital: administration of interacting co-medication and QT prolongation. Eur. J. Clin. Pharmacol. 2016; 72(7): 859-67.

16. ZHU Suyan, LIU Yao, CHEN Chunyan, DING Xiongfang, XU Ping. Anti-SARS-CoV-2 Drugs Induced QT Interval Prolongation: A Literature Review and Pharmaceutical Care.Chin J Mod Appl Pharm, 2020; 37(5):560-3. (In Chinese) 